\title{
Lessons learned: new insights on the role of cytokines in COVID-19
}

\author{
In the midst of resurging COVID-19 cases, the second NIH/FDA virtual COVID-19 and Cytokines symposium was held \\ on 1 December 2020, focusing on longitudinal studies of COVID-19 immunity, including long-term consequences, \\ potential associations with autoimmunity and the multisystem inflammatory syndrome in children (MIS-C).
}

$\Lambda$ central and ongoing quest in COVID-19 research is to establish why and how SARS-CoV-2

elicits heterogeneity in disease severity and immunopathology among infected individuals. Hence, much effort has been exerted to understand the cellular basis of SARS-CoV-2-induced immune responses, with the aim of identifying new biomarkers and prognostic tools and developing new therapeutic options. Cytokines emerged early as critical parameters in COVID-19 disease progression, and understanding the qualitative, quantitative and temporal differences in cytokine expression is considered critical for the conquest of COVID-19.

As the late-2020 fall surge brought the third phase of the COVID-19 pandemic, with record numbers of new cases and deaths, the NIH/FDA Immunology, COVID-19, and Cytokine Interest Groups hosted the second NIH/FDA virtual COVID-19 and Cytokines symposium, bringing together experts in these areas to present the most up-to-date data and to provide a forum for discussion, which focused on recent immunological characterization of the disease and its consequences, including MIS-C.

Opening with a summary of the current challenges and achievements in fighting SARS-CoV-2, Anthony Fauci (NIAID/NIH) and Janet Woodcock (CDER/FDA) presented a road map for extending these efforts, as our understanding of the disease and tools for managing COVID-19 are evolving ${ }^{1}$. Fauci reflected on parallels with the human immunodeficiency virus (HIV) epidemic in the 1980s, which revealed gaps in our knowledge but also led to many outstanding basic and clinical discoveries. Similarly, COVID-19 is an enormous public health problem that challenges scientists and clinicians to rapidly advance our understanding of the immune system and its impact on human disease. Fauci further emphasized the importance of delineating the role of cytokines in COVID19 pathogenesis and resolution and the effects of antiviral and immunomodulatory treatment strategies, as well as vaccines, on immune responses. In turn, Woodcock pointed out that therapeutic interventions serve as a learning tool and can provide new insights into the pathogenesis of the disease. However, the lack of markers defining different stages of the disease has impeded our understanding of the proper timing for treatment regimens, with some therapies resulting in negative effects when given too late or too early in the disease course. The work presented in this symposium outlined efforts to address these issues.

\section{Cellular origins of cytokines in COVID-19}

Adrian Hayday (Crick Institute and King's College) led the meeting by presenting the results of a comprehensive analysis of 63 patients with COVID-19 who were segregated into three groups on the basis of their disease severity. Patients were listed as 'low' when mildly symptomatic (WHO scores 1-2), 'moderate' when little or no supplemental oxygen was required (WHO scores 3-4) and 'severe' when they showed any of the following indications: high-flow oxygen requirement, mechanical ventilation, multi-organ support or death (WHO scores 5-8). At the same time, 55 healthy adult volunteers, of whom 23 had previously experienced SARS-CoV-2 infection without hospitalization, served as healthy controls. Approximately 600 data points were collected from an initial and then two subsequent blood samplings, and these data included complete serology, immune cell analysis and, importantly, cytokine expression (data are publicly available at COVIDIP). Principal component analyses of the datasets showed a clear segregation of COVID-19 samples from control samples, but no segregation of seropositive and seronegative healthy controls ${ }^{2}$. Thus, COVID-19 imposes a distinct immune phenotype. A striking feature of immune responses associated with severe COVID-19 turned out to be the loss of plasmacytoid dendritic cells and basophils, which is accompanied by extreme $\mathrm{T}$ cell cytopenia, which mainly manifests as $\mathrm{CD} 8^{+} \mathrm{T}$ and $\gamma \delta \mathrm{T}$ cell depletion, even though $\mathrm{T}$ cells are not targeted by SARS-CoV-2. Hayday reminded us that isolated parameters rarely provide immunological answers to viral infections and that, in fact, the correlations between the parameters provide more insight about a given pathology. As such, it was interesting that the expression of a triad of cytokines, comprising interleukin (IL)-6, IL-10 and interferon (IFN)- $\boldsymbol{\gamma}$-induced protein 10 (IP-10), is closely correlated with disease progression. IP-10 (CXCL10) is of particular interest because its expression pattern in patients with COVID-19 is distinct from that observed with conventional viral infections. While IP-10 is rapidly but transiently induced in common cold viral infections, IP-10 concentrations frequently remain elevated throughout the COVID-19 response. Moreover, a decline in the level of serum IFN- $\gamma$, a strong inducer of IP-10 expression, did not affect IP-10 concentrations. These findings suggest that IP-10 expression in COVID-19 is maintained independently of IFN- $\gamma$, albeit in a virus-dependent manner. This distinct cytokine profile was confirmed in an expanded study with 305 samples, whereby the rise in cytokine production correlated prognostically with deterioration of patient health status. Notably, the expression of IP-10, together with IL-6 and IL-10, was found to persist with COVID-19 for over 72 hours after admission. Elevated IP-10 was previously reported for SARS-CoV and Middle East respiratory syndrome coronavirus (MERS-CoV) infections, so these features may represent an immune signature of severe coronavirus infection.

Identifying the distinct cytokine profile and immune signature of COVID-19 has practical implications, as it can be prognostic of clinical deterioration, such as requiring intubation or mortality. This led to the important question of whether such distinct immune profiles are only correlated with symptomatic SARS-CoV-2 infection or whether they are also associated with asymptomatic individuals with SARS-CoV-2. To address this question, Nina Le Bert (Duke-NUS Medical School) studied a cohort of patients in Singapore. Here, she asked whether the 


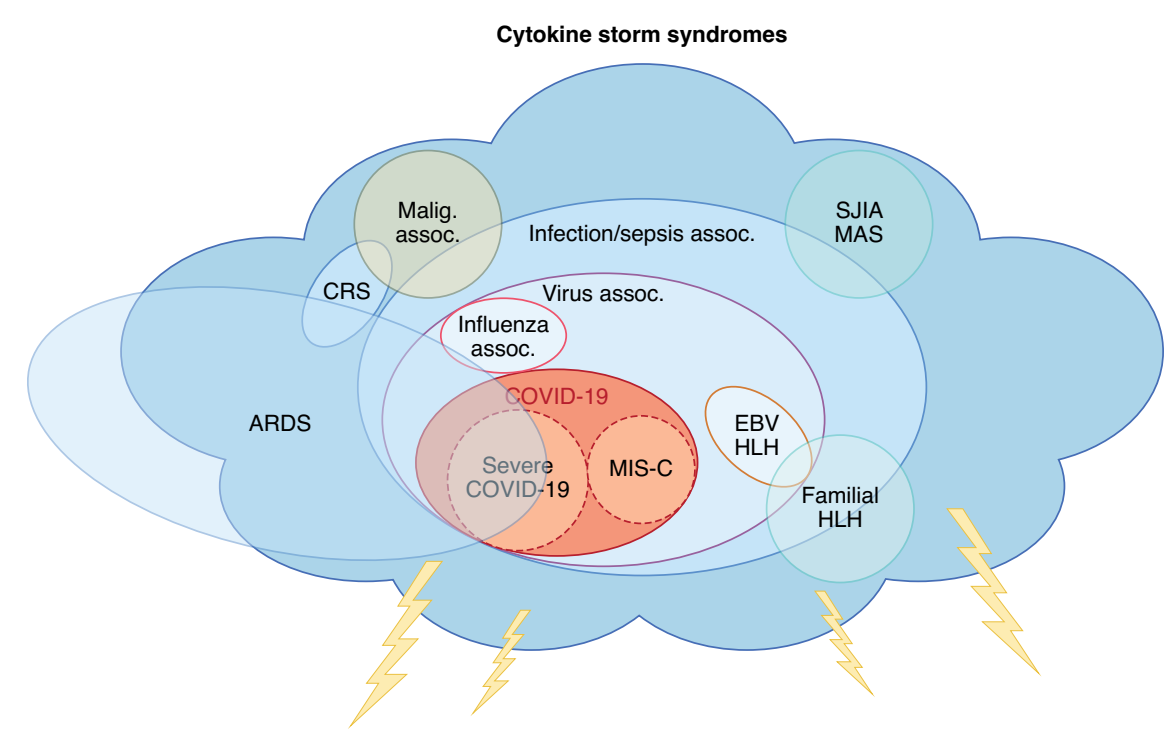

Fig. 1 | The family of disease states that fall under the umbrella term 'cytokine storm'. The disease phenotype of COVID-19 resembles that of other pathological syndromes that are driven by uncontrolled immune system activation and cause cytokine storm. The consequent inflammation can lead to tissue and organ damage. COVID-19 has overlap with features of acute respiratory distress syndrome (ARDS) and post-viral syndromes. MIS-C has less lung involvement (ARDS) and more features of Kawasaki disease. Assoc., association; CRS, cytokine release syndrome; EBV, Epstein-Barr virus; HLH, hemophagocytic lymphohistiocytosis; Malig., malignancy; MAS, macrophage activation syndrome; SJIA, systemic juvenile idiopathic arthritis.

T cell immune responses in symptomatic versus asymptomatic COVID-19 differ and whether disease severity would vary depending on the quality of the immune response. Importantly, COVID-19 cases in Singapore represented a unique chance to assess an understudied population, as many patients are migrant workers, who mostly reside in crowded dormitories. Among the initial 541 recruits, 478 patients underwent the initial assessment as well as 2-week and 6-week follow-up exams. This cohort was then divided on the basis of COVID-19 symptoms: 44 individuals were symptomatic, and the rest of the individuals who tested positive $(90.8 \%)$ were asymptomatic. One of the first questions was whether asymptomatic patients would also mount productive immune responses to SARS-CoV-2. The answer was a resounding yes: anti-nucleoprotein IgG and neutralizing antibody titers were comparable to those of symptomatic patients. Titers also increased with time (measured at 2 and 6 weeks after the initial assessment), indicating a robust humoral immune response regardless of symptoms. To examine whether there would be qualitative differences in the $\mathrm{T}$ cell responses between the two groups, Le Bert and colleagues utilized a pool of overlapping peptides derived from the SARS-CoV-2 membrane $(\mathrm{M})$, nucleoprotein $(\mathrm{NP})$ and spike (S) proteins and challenged T cells from both symptomatic and asymptomatic individuals. Both the strength and antigen specificity of $\mathrm{T}$ cell responses showed no major differences, indicating that the magnitude of $\mathrm{B}$ or $\mathrm{T}$ cell reactivity did not correlate with disease outcome. Next, whole blood cells were stimulated with pools of 15-mer peptides from structural proteins of SARS-CoV-2 and were assessed for cytokine expression. The results demonstrated that both symptomatic and asymptomatic patients mounted strong cytokine responses but that they segregated into distinct clusters, wherein asymptomatic individuals showed high expression of IL-2 and IFN- $\gamma$ while symptomatic individuals showed low expression of IL- 2 and IFN- $\gamma$. Collectively, Le Bert's data, as well as data from Hayday, indicated that a combination of parameters can segregate patients and reflect their condition.

Another important but poorly understood parameter is the contribution of host factors to disease development and progression. In this regard, Raphaela Goldbach-Mansky (NIAID, NIH) summarized her efforts to identify the distinct immunological features of SARS-CoV-2 infection in the context of the host inflammatory response in patients with severe disease. Whether the inflammatory responses in COVID-19 replicate those in autoinflammatory diseases or whether there are unique features in SARS-CoV-2 infection is not clear. In a joint effort with Luigi Notarangelo and Helen Su (both from NIAID), Goldbach-Mansky analyzed a cohort of critically ill patients from Northern Italy, focusing on changes seen in inflammasomopathies and interferonopathies. While autoinflammatory diseases such as the macrophage activation syndrome (MAS) or secondary hemophagocytic lymphohistocytosis (HLH) trigger ultra-high concentrations of IL-18, COVID-19 elicited only modest concentrations of this cytokine (Fig. 1). Also, COVID-19 was rarely associated with thrombocytopenia or hypertriglyceridemia, which is distinct from what is observed with MAS. Instead, patients with COVID19 displayed features of strongly activated cytokine/chemokine secretion pathways that are associated with NF- $\mathrm{\kappa B}$ activation and type II IFN expression. Conversely, the antiviral pathway in COVID-19 appears to be paralyzed, as demonstrated by weak type I IFN responses. Thus, the loss of the type I IFN response can be considered an immune signature of COVID-19. In fact, genetic variants affecting type I-IFNs ${ }^{3}$ or the surprising presence of neutralizing type I IFN autoantibodies ${ }^{4}$ correlate with disease severity in some patients with COVID-19.

Along these lines, Alessandro Sette (La Jolla Institute for Immunology) asked whether there is pre-existing immunity to SARS-CoV-2 in unexposed individuals and, if so, how this could affect disease progression. To understand the adaptive immune response to SARS-CoV-2, Sette's group ventured to map the $\mathrm{T}$ cell epitopes in convalescent patients with COVID-19 and healthy unexposed controls ${ }^{5}$. Surprisingly, SARS-CoV-2-reactive $\mathrm{CD}^{+}{ }^{+} \mathrm{T}$ cells were already present at significant frequencies $(\sim 40-60 \%)$ in unexposed (obtained before the emergence of SARS-CoV-2) individuals. Epitope mapping further revealed that these T cells (1) were mostly reactive to non-spike proteins, (2) displayed much greater responsiveness to peptides from the homologous regions of the common cold coronaviruses (CCC) than to SARS-CoV-2 peptides and (3) were mostly memory CD4 ${ }^{+} \mathrm{T}$ cells. Thus, these results strongly suggested that there is pre-existing immunity to SARS-CoV-2 in healthy donors, which was acquired from past infections with CCC. Whether such cross-reactive antiviral immunity provides protection against COVID-19 remains to be examined. However, it is likely that pre-existing $\mathrm{T}$ cell immunity would only lessen the severity of the disease rather than 
prevent the infection itself. In support of this hypothesis, $\mathrm{T}$ cell epitope mapping in convalescent individuals revealed that the repertoire significantly differed from that in unexposed individuals. In-depth analysis of $\mathrm{T}$ cell epitopes from 100 convalescent individuals further identified a total of 280 major histocompatibility complex (MHC) class II epitopes. These important data revealed that the receptor-binding domain of SARS-CoV-2 spike protein is a weak CD4 ${ }^{+}$ $T$ cell antigenic determinant and that 15-20 different epitopes are recognized by each individual. For $\mathrm{CD}^{+} \mathrm{T}$ cells, a total of 523 MHC class I epitopes were identified, which unveiled an interesting dichotomy between the frequency and reactivity of the human leukocyte antigen haplotype. As such, less frequent HLA-A ${ }^{\star} 0101$-associated epitopes showed a more vigorous response than HLA-A ${ }^{\star} 2601-$ and HLA-A ${ }^{\star} 6801$-associated epitopes. Altogether, these results documented a COVID-19-specific signature in the $\mathrm{T}$ cell immune response that is distinct from the response to CCC, and epitope mapping further showed that HLA binding is the main determinant of the immunogenicity of SARS-CoV-2.

\section{Multisystem inflammatory syndrome in children}

Despite initial optimism that children were spared from the worst effects of COVID-19 (ref. ${ }^{6}$ ), it soon became clear that children could not only develop severe COVID-19 but also a rare secondary inflammatory syndrome, now known as MIS-C. However, the molecular basis of MIS-C and its pathogenesis remain poorly understood. Randy Cron (University of Alabama at Birmingham) started the second session by providing a comprehensive overview of the history, clinical presentation and treatment of this syndrome (Fig. 2). MIS-C was first identified when the pandemic moved to Europe and reports from Northern Italy ${ }^{7}$ and then the $\mathrm{UK}^{8}$ noted an approximately 30 -fold increase in what was thought to be Kawasaki disease (KD), an inflammatory syndrome in children associated with fever, rash, mucocutaneous inflammation and vasculitis, particularly of cardiac arteries. However, these patients were generally more severely ill and had positive serology for SARS-CoV-2, indicative of previous infection or exposure and thus suggesting a distinct entity. MIS-C has many overlapping features with $\mathrm{KD}$ and toxic shock syndrome, but patients are often older than those with $\mathrm{KD}$, which usually occurs in children under the age of five. A similar post-COVID-19 inflammatory syndrome is also observed in young adults but with more evidence of carditis, including cardiac dysfunction, shock and neurological involvement. Notably, patients with MIS-C have more pronounced lymphopenia, anemia and thrombocytopenia as compared to patients with $\mathrm{KD}$. In addition to high C-reactive protein (CRP) levels, troponins and $\mathrm{N}$-terminal pro-B-type natriuretic peptide were specifically elevated, indicative of cardiac involvement ${ }^{8}$. As the pandemic spread to the US, reports of a similar disease in New York and other states ${ }^{9,10}$ noted high frequencies of gastrointestinal (GI) and hematological features. The peak of MIS-C in affected geographical regions generally occurs a month or more after the peak incidence of COVID-19 infections.

Cron proposed that $\mathrm{KD}$ is probably a syndrome with multiple triggers (including a previous infection with another coronavirus) and proposed that MIS-C and severe COVID-19 infection be included under the umbrella of similar cytokine storm syndromes (Fig. 1) ${ }^{11}$. He described studies on the immunological effects of MIS- $\mathrm{C}^{12,13}$, which included lymphopenia, thrombocytopenia and elevated serum IFN- $\gamma$, IL-1 $\beta$, IL-6, IL-10 and IL-17 concentrations, although not to the same extent as in the more severe cytokine storm syndromes. Elevated concentrations of IL-10 correlated with a lower viral load ${ }^{13}$, and its presence together with elevated concentrations of tumor necrosis factor (TNF) was the best marker to distinguish MIS-C from severe COVID-19. Notably, unlike severe COVID-19, MIS-C is not associated with pre-existing comorbidities, with the occasional exception of asthma. Cron outlined treatment options, which included immunomodulatory agents such as intravenous immunoglobulin infusions, as used for KD, glucocorticoids, anakinra (IL-1 receptor antagonist) and infliximab (a monoclonal antibody to TNF); antimicrobials, including remdesivir and broad-spectrum antibiotics (as some patients' clinical pictures resemble bacterially induced toxic shock syndrome); and, in severe cases, mechanical ventilation, pressor support and anticoagulants $s^{8,9,14,15}$. Updated treatment guidelines were published recently by the American College of Rheumatology ${ }^{16}$.

To investigate MIS-C from a systems biology perspective, similar to studying COVID-19 in adults, Dusan Bogunovic (Icahn School of Medicine at Mount Sinai) described analyses of about 40 patients treated at Mount Sinai in New York who presented with the symptoms of MIS-C and who met diagnostic criteria ${ }^{16}$. Treatment with an IL-6R blocker and intravenous immunoglobulin led to discharge, usually within 5 days, with universally favorable outcomes. To provide molecular insight into the disease, they combined serology, cytokine profiling, high-throughput 85-parameter immunophenotyping and autoantibody analysis for nine of these patients ${ }^{17}$. MIS-C SARS-CoV-2 antibody responses resembled those seen in convalescence, including effective virus neutralization in vitro, but with lower IgM and higher IgA concentrations. Multiplex cytokine array data revealed a unique MIS-C inflammatory cytokine signature not seen in healthy controls or patients with COVID19, which included the expression of chemokines (CXCL1, CXCL6 and CXCL11, as well as CCL3 and CCL19) and cytokines (including IL-6 and IL-17A, as noted by Cron) involved in myeloid cell chemotaxis and inflammation. In parallel, mass cytometry immunophenotyping suggested immune cell activation and egress of natural killer (NK) and myeloid cells to peripheral organs. Autoantibody analysis indicated the presence of both autoantibodies targeting organs central to MIS-C pathology, including the GI tract, immune cells and cardiac and epithelial tissue, and some previously described autoantigens. Interestingly, pathways involved in the sensory detection of smell were also targeted by autoantibodies. Using cellular indexing of transcriptomes and epitopes by sequencing (CITE-seq) to simultaneously detect surface antigens and perform single-cell RNA sequencing (scRNA-seq), Bogunovic and his group found increased expression of genes involved in lymphocyte activation and cytotoxicity of $\mathrm{NK}$ and $\mathrm{CD} 8^{+} \mathrm{T}$ cells. These findings were particularly pronounced in a subset of patients who showed increased phosphorylation of the signaling molecule STAT3 across cell types.

To further address the molecular mechanisms of MIS-C immunopathology, Carrie Lucas (Yale School of Medicine) focused on two potential causes of post-COVID-19 MIS-C: (1) a rare second hit with a pathogen within a certain window of time after SARS-CoV-2 infection and (2) a unique immune response to SARS-CoV-2 that triggers a transient reactivity against the self. Lucas presented her team's recent work on a cohort of 15 patients who developed MIS-C approximately 1 month after a peak of COVID-19, whom they stratified into two groups according to disease severity (patients with severe disease were those requiring ventilatory support or pressors) (Fig. 2) ${ }^{18}$. She described CITE-seq results, including $\mathrm{T}$ cell antigen receptor (TCR) and $B$ cell antigen receptor (BCR) repertoire analyses, for peripheral blood mononuclear cells from seven patients with MIS-C, as well as from pediatric and adult patients 


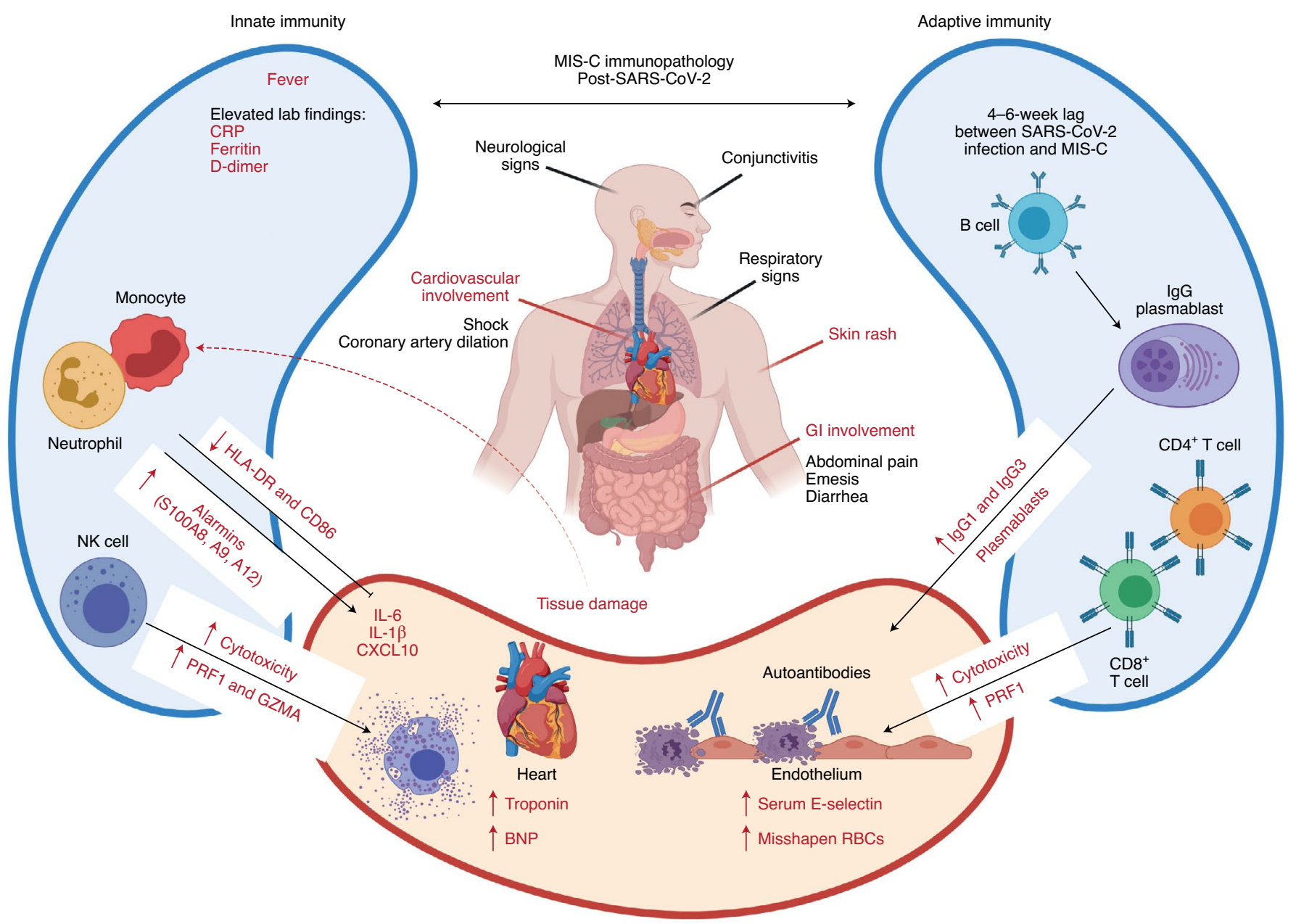

Fig. 2 | Immunopathology associated with MIS-C post-SARS-CoV-2. MIS-C is characterized by fever and cardiovascular, GI tract, neurological, respiratory and mucocutaneous inflammation. Immunological features include activation of both innate and adaptive responses, including elevated expression of genes encoding S100 alarmins and increased concentrations of acute phase proteins (CRP, ferritin and D-dimers) as well as IL-6, IL-1 $\beta$ and CXCL10, indicative of systemic inflammation. Activation of $\mathrm{NK}$ and $\mathrm{CD} 8^{+} \mathrm{T}$ cell cytotoxicity probably contributes to inflammation, as well as increased numbers of proliferating plasmablasts, which may produce autoreactive IgGs. Endothelial damage is evidenced by elevated concentrations of soluble E-selectin in the serum and cardiac damage by increased amounts of troponin and $\mathrm{N}$-terminal pro-B-type natriuretic peptide (BNP). Figure adapted with permission from ref. ${ }^{18}$, Cold Spring Harbor Laboratory; made in (CBioRender-biorender.com.

with COVID-19 and controls. Evaluation of transcriptional responses revealed that antirespiratory virus and antibacterial response signatures were low in patients with MIS-C, confirming that SARS-CoV-2 infection was cleared, with no evidence of secondary infection. Moreover, no reads were present for Epstein-Barr virus or cytomegalovirus. In parallel, serum proteomics confirmed elevated proinflammatory cytokines and chemokines involved in myeloid cell infiltration into the tissues, similar to the findings from Bogunovic. Looking for the drivers of this hyperinflammatory phenotype, they analyzed gene expression in different cell types and found that the expression of genes encoding S100A alarmins was elevated (similar to results in a recent report on
COVID-19 by Guo et al. ${ }^{19}$ ) and class II HLA and CD86 were downregulated in myeloid cells. Again, in NK cells and CD8 ${ }^{+} \mathrm{T}$ cells, cytotoxicity genes (encoding perforin and granzymes $\mathrm{A}$ and $\mathrm{H}$ ) and IL-32 were elevated (Fig. 2). Lucas also noted increased numbers of plasmablasts in patients with MIS-C, which were enriched for IgG1 or IgG3 isotypes and correlated with proliferating $\mathrm{CD}^{+} \mathrm{T}$ cells with a B helper gene profile. Patients with severe MIS-C exhibited less TCR and BCR diversity, elevated numbers of plasmablasts and greater somatic hypermutation as compared to the moderate disease group. The patients with severe disease also exhibited elevated concentrations of serum soluble E-selectin, also seen in $\mathrm{KD}$, suggesting endothelial damage; functional experiments provided evidence for serum IgG antibodies recognizing cultured endothelial cells.

Together with the work of Bogunovic, these studies provide insight into immunological signatures of MIS-C, which, along with functional studies and a potential mouse model, may help predict, prevent and treat MIS-C post-SARS-CoV-2 infection in children. Nonetheless, many questions remain, including whether there is a specific trigger for MIS-C and whether there are contributions from the gut commensal microbiota or pathogens, associations with food, or oral routes of transmission, given the involvement of the GI tract. Furthermore, although there was no evidence of secondary respiratory infection as a MIS-C trigger, tissue-specific secondary infection or damage (such as 
from gut infections) or the involvement of superantigens, a topic that was addressed in the next talk, could not be ruled out.

\section{Autoimmunity and other long-term consequences of COVID-19}

One of the most notable clinical outcomes of SARS-CoV-2 is the induction of autoimmune disease that persists long after the resolution of acute viral infection in a subpopulation of patients. This topic was the focus of the talks from Alessio Fasano (Massachusetts General Hospital), Virginia Pascual (Weill Cornell Medicine) and Leonard Calabrese (Cleveland Clinic), who presented different aspects of the intersection between COVID-19 and autoimmunity. Fasano opened the session by conveying data from several studies performed in Italy suggesting that the prevalence and severity of COVID-19 in patients with autoimmune diseases (AIDs) is similar to those of the general population $^{20}$. However, symptoms consistent with immune thrombocytopenic purpura (ITP), Miller Fisher syndrome, GuillainBarré Syndrome (GBS), anti-phospholipid syndromes (APLS) and MIS-C have been described in patients following SARS-CoV-2 infection, leading his group to focus on the molecular mechanisms that make the development of AIDs more likely in patients with COVID-19 patients. His talk centered on three principal hypotheses ${ }^{21}$ underlying the induction of autoimmunity by severe viral infection: molecular mimicry, viral and bacterial superantigens altering the $\mathrm{T}$ cell repertoire, and lymphocyte apoptosis followed by expansion of autoreactive lymphocytes. Regarding molecular mimicry, he remarked on multiple shared motifs between the spike protein and human chaperone proteins and hypothesized that altered processing and presentation of endogenous antigens could foster the recognition of self-antigens. He also hypothesized that the loss of tolerance associated with lymphocyte apoptosis during acute infection followed by a skewed expansion of the repertoire could be involved, leading eventually to autoimmunity, as proposed by Canas et $\mathrm{al}^{22}$. However, Fasano considered the strongest mechanistic trigger for the hyperinflammatory syndrome and AID induction to be viral superantigens within SARS-CoV-2, which induce non-specific activation and expansion of a distinct population of T cells with a skewed TCR repertoire, which leads to the expansion of plasmablasts ${ }^{23}$. In particular, the expansion of TCRBV11-2-bearing T cells appeared to correlate with elevated proinflammatory cytokines associated with cytokine storm in children with MIS-C ${ }^{24}$. Lastly, drawing from the parallels between MIS-C and $\mathrm{KD}$, Fasano described a potential role for zonulin-dependent increased permeability of the intestinal epithelial barrier and proposed a provocative hypothesis wherein the viral infection results in intestinal dysbiosis (pertaining to altered gut microbiota composition and function), which then triggers MyD88-dependent zonulin release. Once released, zonulin binds to the proteinase-activated receptor 2 , triggering a sequence of signaling events that ultimately cause disassembly of tight junctional complexes and increased GI mucosal permeability. This increased mucosal permeability permits viral or even bacterial antigens to be internalized and act as superantigens, thus triggering a cytokine storm and autoimmunity (Fig. 3).

The autoimmune component of COVID-19 was also explored by Pascual, who described multiple studies indicating parallels between COVID-19 and autoimmune disorders. After describing the clinical features shared by COVID-19 and AIDs, she focused on mechanistic commonalities, particularly the central role of type I IFNs. Citing findings published by Casanova, $\mathrm{Su}$ and others regarding the increased severity of COVID-19 in patients with reduced IFN function due to inborn errors in type I IFN immunity or autoantibodies to type I IFNs ${ }^{3,4}$, she emphasized the critical role of type I IFN in controlling viral load. At the same time, she remarked that the pattern of IFN-inducible genes expressed following a viral infection is similar to that in systemic lupus erythematosus (SLE) and a number of monogenic interferonopathies described in children, as well as in subsets of patients with other AIDs such as Sjogren's, dermatomyositis, thyroiditis and diabetes, suggesting that it may underlie some of the clinical features. Citing results from Goldbach-Mansky and Notarangelo that showed a 10-fold increase in type I IFNs as well as an association between CXCL10 and COVID-19 severity, Pascual remarked that, although the expression of interferon-inducible genes was lower in patients with COVID-19 than in children with interferonopathies or pediatric SLE, it was in line with that observed in adults with $\mathrm{SLE}^{25}$, leading her to emphasize the different type I IFN responses in pediatric and adult patients. In support of an autoimmune component for COVID-19, Pascual also remarked on the presence of antibodies to IFNs, coagulation factors, phospholipids and nucleic acids in patients with COVID-19, which are characteristic of several autoimmune disorders, signaling the need to evaluate the long-term persistence and potential sequelae of these antibodies in patients that survive COVID-19. Pascual, then focused on the emergence of autoreactive $\mathrm{T}$ and $\mathrm{B}$ cells, again drawing interesting parallels between the adaptive immune responses in patients with COVID19 and pediatric patients with SLE. She pointed to studies by Ignacio Sanz that showed a potential role for the extrafollicular activation of autoreactive $B$ cells in patients with severe COVID-19, drawing parallels with SLE and underscoring a potential role for a subpopulation of memory B cells that lack follicular and conventional memory markers but express Toll-like receptor 7 (TLR7) and antigen-presenting markers, also seen in the peripheral blood of patients with $\mathrm{SLE}^{26-28}$. She further proposed that the surge in these extrafollicular plasmablasts is possibly helped by extrafollicular proinflammatory T cells displaying exhaustion/hyperproliferative and cytotoxic markers, as recently described for severe COVID-19 (refs. ${ }^{29,30}$ ). Interestingly, CD4 ${ }^{+}$ $\mathrm{T}$ cells with similar characteristics that lack follicular markers are also reported in children with SLE, reinforcing the concept that the dysfunctional immune response in severe COVID-19 resembles that found in SLE. Pascual then proposed a model wherein virus-activated plasmacytoid dendritic cells ( $\mathrm{pDCs}$ ) produce type I IFN that primes $\mathrm{CD}^{+} \mathrm{T}$ cells to provide help to extrafollicular B cells in unconventional ways, as they do in $\mathrm{SLE}^{31}$. While the cellular source of type I IFN has not been confirmed, given the reported reduction in blood pDCs in patients with COVID-19 (ref. ${ }^{17}$ ) and SLE, she speculated that this could correspond to increased migration of pDCs to inflamed tissues ${ }^{32}$. Pascual's talk stressed that mechanistic understanding of autoimmune diseases may help to decipher the pathogenicity of COVID-19 and identify therapeutic targets. In turn, detailed longitudinal studies of patients with COVID-19 may shed light on pathogenic autoimmune mechanisms and the potential for immunomodulatory therapeutics to mitigate autoimmune diseases.

The theme of shared mechanisms between AIDs and COVID-19 was picked up by Leonard Calabrese, who closed the session by asking two questions: what can we learn about cytokines in COVID-19 by examining the outcomes for individuals with pre-existing autoimmune/autoinflammatory disorders who develop COVID-19, and what effects do the commonly used therapies for such autoimmune diseases, including immune-pathway-targeted biologics and non-biological disease-modifying 


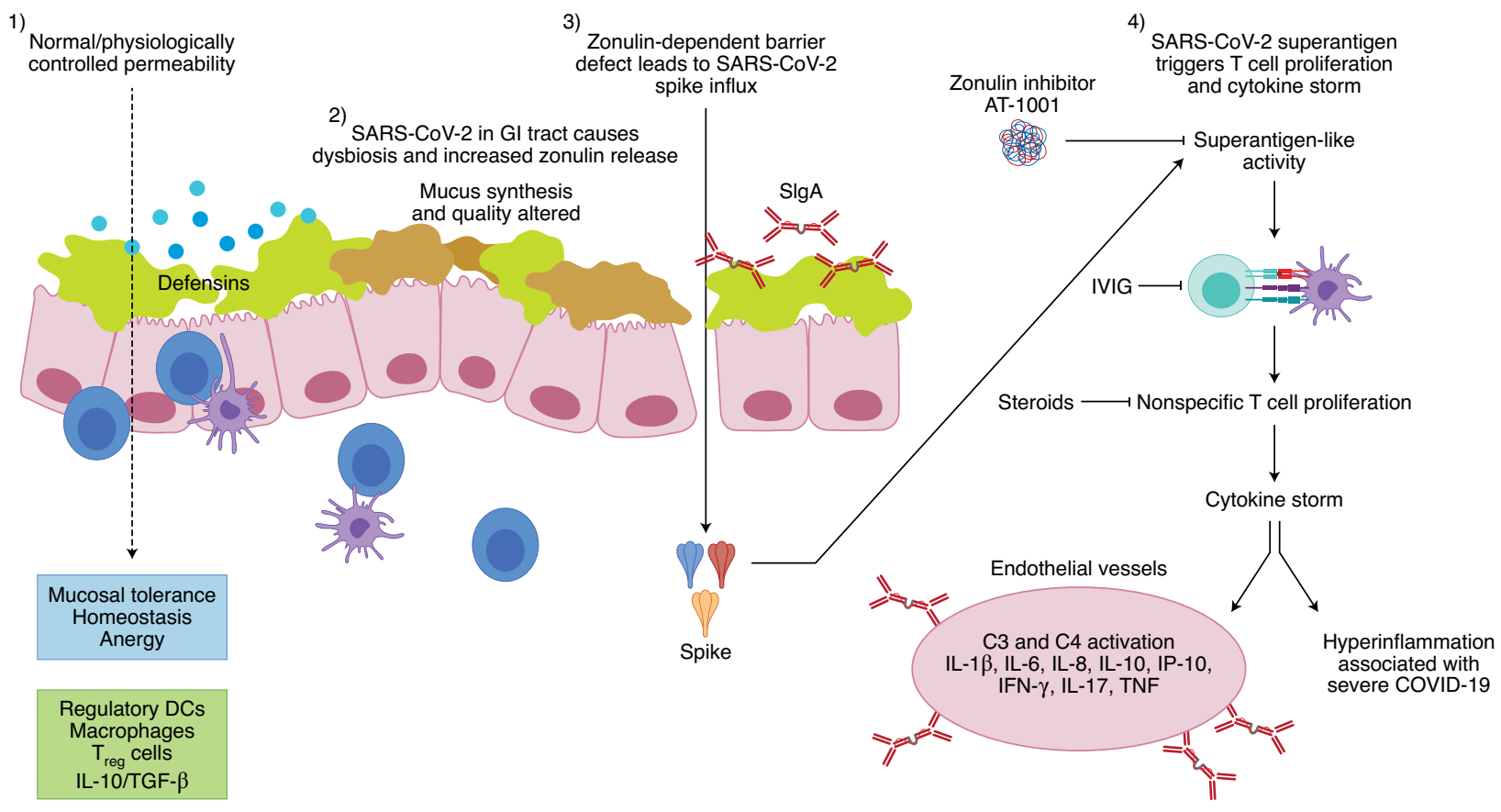

Fig. 3 | Hypothesis on the pathogenesis of multisystem inflammatory syndrome in children and in adults (MIS-A). (1) Under physiological circumstances, the homeostasis of the intestinal mucosa is maintained through tightly controlled antigen trafficking from the gut lumen into the lamina propria. (2) The presence of SARS-CoV-2 (either as viable or non-viable viruses) in the GI tract causes dysbiosis and upregulates the expression of zonulin, a molecule that controls paracellular permeability. (3) The zonulin-dependent increased gut permeability allows the paracellular passage of large, intact molecules, including SARS-CoV-2-derived spike proteins, into the lamina propria. (4) Spike proteins, acting as superantigens, trigger T cell proliferation and cytokine storm, with subsequent onsets of severe GI symptoms and systemic inflammation, which are typical of MIS-C and MIS-A. IVIG, intravenous immunoglobulin. SIgA, secretory immunoglobulin A.

anti-rheumatic drugs (DMARDs), have on the clinical outcome of COVID-19?

He consulted three patient registries to attempt to answer these critical questions: the COVID-19 Global Rheumatology Alliance (GRA), consisting of the Global UCSF Registry (3,572 patients) and the European/EULAR registry (3,239 patients), representing the largest database; the Secure-IBD Database on COVID-19 (based at the University of North Carolina at Chapel Hill), a web-based voluntary reporting system in which health care providers report confirmed COVID-19 cases and patient outcomes along with medication exposure data; and the Psoriasis Registry for Outcomes, Therapy and epidemiology of COVID-19 infection (PsoProtect), containing 557 cases (at the time of reporting).

Calabrese pointed out that these registries are imperfect for evaluating the effects of underlying disease per se, as each may impact COVID-19 prognosis, given the markedly disparate and heterogeneous diseases in these databases. However, the effect of baseline therapies and risk factors for severe disease or death were still critically analyzed despite important limitations regarding selection bias, low granularity, lack of control comparators and residual confounding factors in such registries. He repeatedly emphasized that such studies were important for hypothesis generation rather than for producing conclusive findings.

Initially reported at the American College of Rheumatology meeting in November 2020, data were also presented at this meeting that examined risk factors associated with death in the GRA database from a population of over 4,000 patients. Adjusting for multiple variables, the following were associated with an increased risk of death from COVID-19 (odds ratio > 1): no DMARDs, sulfasalazine, rituximab, and glucocorticoids at a dose of $>10 \mathrm{mg}$ per day. While rituximab's risk stems from the failure to generate humoral immunity (and possibly $\mathrm{T}$ cell immunity due to the lack of $B$ cell antigen presentation), and glucocorticoids at the higher dose potently suppress antiviral responses, the mechanism by which the absence of DMARDs and sulfasalazine contribute to enhanced risk is less clear. The risk pertaining to glucocorticoids was also identified in the SECURE-IBD database, and a multivariate analysis (controlling for age, sex, disease type, disease activity, comorbidities, smoking, obesity, aspirin, and anti-TNF medication) revealed an odds ratio of 6.87 for patients on higher doses of steroids at the time of contracting COVID-19. Similar results were found in other IBD databases from Italy and the US. Nonetheless, the need to understand the context of glucocorticoid administration was emphasized; while dexamethasone was associated with poorer outcomes for patients treated with higher doses of glucocorticoids at the time of acquiring COVID-19 and for patients with mild to moderate disease (no oxygen required), it reduced deaths by one third for patients receiving invasive mechanical ventilation, and it is now the standard of care (RECOVERY trial).

Calabrese then discussed the significance of elevated inflammatory cytokines and the effects of their blockade on the outcome of COVID-19. He pointed to the importance of TNF concentrations and downstream IL-6 and IL-8 concentrations as directly correlated with poor outcomes, including hospitalization, transfer to an intensive care unit, mechanical ventilation and death ${ }^{33,34}$. He also emphasized the contribution of TNF to diverse phenomena affecting COVID-19, including neutrophil activation and the release of neutrophil extracellular traps (further inducing epithelial cell death), 
the loss of germinal centers and induction of extrafollicular B cell responses, as well as the generation of autoantibodies, including phospholipid antibodies ${ }^{33}$. In this context, he discussed analyses of the registries to determine the effects of TNF blockers on COVID-19. In both the GRA and PsoProtect databases, treatment with TNF inhibitors was associated with a favorable outcome, with an odds ratio of 0.4 in the in the GRA database (for hospitalization) and 0.60 in the PsoProtect database (for intensive care unit admission, need for mechanical ventilation, and death). However, in the SECRURE-IBD database, which employed a multivariate analysis, TNF inhibition was associated with a non-significant odds ratio of 0.9 for severe outcomes. It is critical to note, however, that the outcome was not worsened in patients on TNF inhibitors and that such treatment may potentially be beneficial.

The important topic of synergism between cytokines in contributing to the devastating hyperinflammation associated with severe COVID-19 and whether inhibition of multiple inflammatory cytokines would improve the outcome was discussed next. Citing work from murine models, Calabrese discussed data showing that TNF and IFN- $\gamma$ can synergize in mediating PANoptosis (pyroptosis, apoptosis and necroptosis), associated with the induction and perpetuation of cytokine storm and cell death that is observed in both sepsis and COVID-19 animal models ${ }^{35}$. Moreover, treatment of murine cytokine storm models, including SARS-CoV-2 infection, HLH and sepsis, with a combination of TNF and IFN- $\gamma$ inhibitors markedly improved the outcome, suggesting that dual blockade as compared to single agents may improve outcomes in patients.

Blockade of IL- 6 or IL-6R is known to improve the outcome in patients with cytokine release syndrome associated with CAR-T cell therapy. However, although serum IL-6 concentrations are correlated with severe outcomes in COVID-19, Calabrese noted that they are more similar to IL-6 concentrations in acute respiratory distress syndrome and sepsis and lower than those in patients with cytokine release in CAR-T cell therapy. Also noted were the disappointing results thus far of IL-6 blockade in numerous randomized clinical trials, several of which have been halted due to futility. In the ACR database, the only one in which IL-6 inhibitors are tracked, patients with connective tissue diseases appeared to have increased risk of death from COVID-19 if treated with IL-6 inhibitors. However, this was based on a small number of patients, with a wide confidence interval.
Nonetheless, Calabrese echoed Woodcock in questioning the timing of IL- 6 blockade administration as a potential key to the lack of efficacy, citing studies in which genetic polymorphisms in the IL-6R gene that reduce IL-6 signaling were shown to be salutary in COVID-19 as well as in cardiovascular disease. For example, the Asp358Ala variant of the IL-6R receptor disrupts IL-6 signaling and is associated with reduced cardiovascular disease frequency in the general population and lower risk of both SARS-CoV-2 infection and hospitalization for COVID-19 (ref. ${ }^{36}$ ). Calabrese concluded that timing may indeed be a key issue in the appropriate use of IL-6 inhibitors and that treatment with such agents at the onset of disease rather than later may have beneficial effects.

The final topic was treatment of COVID-19 with the JAK inhibitor baricitinib. Noting that the ACTT-2 trial of baricitinib and remdesivir versus remdesivir alone had a favorable but very modestly improved outcome for the combination therapy (shortening of clinical disease by only one day), Calabrese stressed that such limited efficacy must be weighed against the potential adverse consequences of baricitinib, including inhibition of numerous cytokines that may play an important role in antiviral responses (type I IFNs, IL-12, and so on), promotion of thromboembolism (already a critical problem in patients with COVID-19) and activation of endogenous viruses, including herpes and $\mathrm{BK}$ viruses. These potential negative effects must be considered carefully against the marginally improved outcome when baricitinib is given in combination with remdesevir, along with the antiviral properties that have been identified for baricitinib ${ }^{37}$. Other considerations regarding this agent include issues of the timing of administration and, critically, how its administration should be considered in view of the known salutary effects of dexamethasone for severe disease.

Finally, in addition to citing the utility of registries for providing insights and for hypothesis generation, Calabrese concluded that the current data support contextual roles for cytokines in disease and their inhibitors in treatment, and these are influenced by disease stage and the associated immunologic 'endotype.' Such considerations should be fundamental in designing new trials. Importantly, all three speakers agreed that the consensus at this time is that infection with SARS-CoV-2 does not lead to flare-ups in patients with AIDs. Of note, Calabrese remarked that many patients had altered their treatment following the onset of the pandemic, complicating the assessment of the consequences of the virus for these patients.

\section{Concluding remarks}

It has become abundantly clear that cytokines play a commanding role in the pathogenesis of COVID-19. Cytokine expression is not only a prognostic biomarker for the disease outcome and severity of COVID-19, but SARS-CoV2 -induced cytokine expression is also understood to be a potent agitator of the host immune system, leading to destructive autoinflammation, organ failure and death. As such, the second NIH/FDA symposium on Cytokines in COVID-19 was timely, showcasing cutting-edge research conducted by the immunology community to understand the cytokine-driven pathology of COVID-19. Harnessing knowledge of the virus as well as of autoimmune processes, the speakers highlighted emerging areas of research that may yield new therapeutic targets to reduce the clinical impact of the disease and minimize sequelae.

The virtual nature of the symposium permitted speakers and audiences from around the world to participate in real time, with over 800 viewers logging in on the day of the event and many more subsequently making use of the archived videos. Such worldwide interest illustrated the cutting-edge nature of the talks but also accentuated the need for rapid dissemination of COVID-19-related data to the scientific community. Nonetheless, it was also clear that many questions remain, including the expression patterns and timing of cytokines that are relevant to the disease course and how differences in genetics and environment may contribute. Furthermore, the many nuances to measuring cytokines, including methods for and timing of the collection, treatment, storage and assay of samples, may need to be addressed before specific cytokines can be used reproducibly as disease biomarkers to monitor and predict disease progression and recovery. SARS-CoV-2 presents a set of unique challenges that require navigation through uncharted territories and collaboration on the part of the scientific community. We expect that increasing efforts to interrogate the role of cytokines in COVID-19 will provides us with new tools to map and understand the molecular landscape of this viral disease.

\footnotetext{
Maja Buszko (D) 1凶, Aleksandra Nita-Lazar², Jung-Hyun Park (D) ${ }^{3}$, Pamela L. Schwartzberg ${ }^{4}$, Daniela Verthelyi (D) ${ }^{5}$, Howard A. Young (D) 6 and Amy S. Rosenberg ${ }^{7}$ ${ }^{1}$ Cellular Immunology Section, Laboratory of Immune System Biology, National Institute of Allergy and Infectious Diseases, NIH, Bethesda, MD, USA.
} 
${ }^{2}$ Functional Cellular Networks Section, Laboratory of Immune System Biology, National Institute of Allergy and Infectious Diseases, NIH, Bethesda, $M D$, USA. ${ }^{3}$ Experimental Immunology Branch, National Cancer Institute, NIH, Bethesda, MD, USA. ${ }^{4}$ Cell Signaling and Immunity Section, Laboratory of Immune System Biology, National Institute of Allergy and Infectious Diseases, NIH, Bethesda, MD, USA. ${ }^{5}$ Laboratory of Innate Immunity, Division of Biotechnology Review and Research-III, Office of Biotechnology Products, Center for Drug Evaluation and Research, U.S. Food and Drug Administration, Silver Spring, MD, USA. ${ }^{6}$ Laboratory of Cancer Immunometabolism, Center for Cancer Research, National Cancer Institute-Frederick, Frederick, MD, USA. ${ }^{7}$ Laboratory of Immunology, Division of Biotechnology Review and Research-III, Office of Biotechnology Products, Center for Drug Evaluation and Research, U.S. Food and Drug Administration, Silver Spring, MD, USA.

$\bigotimes_{e-m a i l: m a j a . b u s z k o @ n i h . g o v}$

Published online: 15 March 2021

https://doi.org/10.1038/s41590-021-00901-9

References

1. Sundararaj Stanleyraj, J. et al. J. Antimicrob. Chemother. 76, 283-285 (2020).
2. Laing, A. G. et al. Nat. Med. 26, 1623-1635 (2020).

3. Zhang, Q. et al. Science https://doi.org/10.1126/science.abd4570 (2020).

4. Bastard, P. et al. Science https://doi.org/10.1126/science.abd4585 (2020).

5. Grifoni, A. et al. Cell 181, 1489-1501.e15 (2020).

6. Lingappan, K., Karmouty-Quintana, H., Davies, J., Akkanti, B. \& Harting, M. T. Am. J. Physiol. Lung Cell. Mol. Physiol. 319, L39-L44 (2020).

7. Verdoni, L. et al. Lancet 395, 1771-1778 (2020).

8. Whittaker, E. et al. JAMA 324, 259-269 (2020).

9. Dufort, E. M. et al. N. Engl. J. Med. 383, 347-358 (2020).

10. Feldstein, L. R. et al. N. Engl. J. Med. 383, 334-346 (2020).

11. Henderson, L. A. et al. Arthritis Rheumatol. 72, 1059-1063 (2020).

12. Lee, P. Y. et al. J. Clin. Invest. 130, 5942-5950 (2020).

13. Diorio, C. et al. J. Clin. Invest. 130, 5967-5975 (2020).

14. Pouletty, M. et al. Ann. Rheum. Dis. 79, 999-1006 (2020).

15. Carter, M. J. et al. Nat. Med. 26, 1701-1707 (2020).

16. Henderson, L. A. et al. Arthritis Rheumatol. https://doi.org/ $10.1002 /$ art.41616 (2020)

17. Gruber, C. N. et al. Cell 183, 982-995.e14 (2020).

18. Ramaswamy, A. et al. Preprint at medRxiv https://doi.org/ $10.1101 / 2020.12 .01 .20241364$ (2020).

19. Guo, Q. et al. Cell Host Microbe 29, 222-235.e4 (2021).

20. Murtas, R. et al. Auto Immun. Highlights 11, 15 (2020).

21. Wucherpfennig, K. W. J. Clin. Invest. 108, 1097-1104 (2001).

22. Cañas, C. A. Med. Hypotheses 145, 110345 (2020).

23. Cheng, M. H. et al. Proc. Natl Acad. Sci. USA 117, 25254-25262 (2020)

24. Porritt, R. A. et al. J. Clin. Invest. (in the press)

25. Abers, M. S. et al. JCI Insight https://doi.org/10.1172/ jci.insight.144455 (2021)

26. Woodruff, M. C. et al. Nat. Immunol. 21, 1506-1516 (2020).

27. Jenks, S. A. et al. Immunity 49, 725-739.e6 (2018).

28. Nehar-Belaid, D. et al. Nat. Immunol. 21, 1094-1106 (2020)
29. Kaneko, N. et al. Cell 183, 143-157.e13 (2020).

30. Su, Y. et al. Cell 183, 1479-1495.e20 (2020).

31. Caielli, S. et al. Nat. Med. 25, 75-81 (2019).

32. Pascual, V., Farkas, L. \& Banchereau, J. Curr. Opin. Immunol. 18, 676-682 (2006)

33. Robinson, P. C., Richards, D., Tanner, H. L. \& Feldmann, M. Lancet Rheumatol. 2, E653-E655 (2020).

34. Del Valle, D. M. et al. Nat. Med. 26, 1636-1643 (2020).

35. Karki, R. et al. Cell 184, 149-168.e17 (2021).

36. Bovijn, J., Lindgren, C. M. \& Holmes, M. V. Lancet Rheumatol. 2 E658-E659 (2020).

37. Stebbing, J. et al. Sci. Adv. 7, eabe4724 (2021).

Acknowledgements

We thank the speakers for their valuable feedback in preparing this Meeting Report. We are also grateful to CBIIT IT operations, NCI, for hosting the virtual conference. Figures were created with the help of the Visual and Medical Arts Unit, Research Technologies Branch, NIAID, NIH, and using Biorender. This work was supported by the Intramural Research Program of the NIH, NIAID; the Intramural Research Program of the Center for Cancer Research, NCI, NIH; and the Office of Biotechnology Products, CDER, FDA. The views expressed in this article are those of the authors and do not necessarily reflect the official policy or position of the United States Food and Drug Administration and the Department of Health and Human Services, nor does mention of trade names, commercial products or organizations imply endorsement by the United States Government.

Competing interests

The authors declare no competing interests. 